\title{
Effect of cylinder proximity to the wall on channel flow heat transfer enhancement
}

\author{
Mohsen Cheraghi*, Mehrdad Raisee, Mostafa Moghaddami \\ Department of Mechanical Engineering, Faculty of Engineering, University of Tehran, P.0. Box 11365/4563, Tehran, Iran
}

\section{A R T I C L E I N F O}

\section{Article history:}

Received 16 July 2013

Accepted 17 December 2013

Available online 24 January 2014

\section{Keywords:}

Vortex shedding

Heat transfer

2D channel

Circular cylinder

Prandtl number

\begin{abstract}
A B S T R A C T
Heat-transfer enhancement in a uniformly heated slot mini-channel due to vortices shed from an adiabatic circular cylinder is numerically investigated. The effects of gap spacing between the cylinder and bottom wall on wall heat transfer and pressure drop are systemically studied. Numerical simulations are performed at $\operatorname{Re}=100,0.1 \leqslant \operatorname{Pr} \leqslant 10$ and a blockage ratio of $D / H=1 / 3$. Results within the thermally developing flow region show heat transfer augmentation compared to the plane channel. It was found that when the obstacle is placed in the middle of the duct, maximum heat transfer enhancement from channel walls is achieved. Displacement of circular cylinder towards the bottom wall leads to the suppression of the vortex shedding, the establishment of a steady flow and a reduction of both wall heat transfer and pressure drop. Performance analysis indicates that the proposed heat transfer enhancement mechanism is beneficial for low-Prandtl-number fluids.
\end{abstract}

(C) 2013 Académie des sciences. Published by Elsevier Masson SAS. All rights reserved.

\section{Introduction}

With the advances in state-of-the-art mini- and micro-scale technologies and demands for higher cooling efficiency in electronic chips, compact heat exchangers and other heat transfer devices, research work on thermal-hydraulic characteristics of mini- and micro-channels is gaining increasing attention. Thermal performance of cooling systems can be improved by different approaches [1-3]. For example, casting artificial roughness or placing obstacles on the solid boundary can substantially increase the wall heat transfer coefficient. Such boundary modifications can generate vortices, which increase mixing, reduce the thickness of the thermal boundary layer, and thereby enhance heat transfer. Since fluid velocities and characteristic length scales are relatively small in mini- and micro-scale devices, the flow Reynolds number is low or moderate. Therefore, these devices are often operating in laminar conditions. Utilizing a proper vortex generation mechanism can effectively enhance heat transfer in such devices. The focus of the present study is to assess how vortices shed from a stationary confined cylindrical obstacle influence thermal and hydraulic characteristics when their position approaches the lower wall.

Heat transfer, both from the channel walls and from the vortex promoter itself, has been the subject of several investigations. For example, Rahnama and Moghaddam [4] studied convective heat transfer over a square cylinder confined in a channel and proposed a correlation between Nusselt and Reynolds numbers. The blockage ratio was $1 / 8$, while the Reynolds number based on the mean flow velocity and chord length of the square cylinder was less than 200 . Their results showed a linear increase in the recirculation length and a decrease in the drag coefficient with increasing the Reynolds number for Reynolds numbers lower than 70. They reported an increase in the Nusselt number and the drag coefficient

\footnotetext{
* Corresponding author.

E-mail address: m.cheraghi@ut.ac.ir (M. Cheraghi).
} 
with a Reynolds number's increase for the unsteady flow regime, where vortex shedding was observed from the cylinder. Heat transfer augmentation and entropy generation were determined experimentally at $R e=3450$ for an air flow through a ribbed duct with a circular vortex generator placed immediately above or just downstream from the selected rib elements by Yrum et al. [5]. Their results showed that the smaller diameter generators have small effects on the Nusselt number and entropy generation. In all cases examined, a generator-induced local Nusselt enhancement occurred in the inter-rib space behind the rib pair, regardless of the generator's diameter. Wang and Jaluria [6] also investigated fluid flow and mixed heat transfer in a grooved channel in the presence of a rectangular obstacle and examined the effect of obstacle's geometry on flow characteristics at $600 \leqslant R e \leqslant 2250$. Their results showed that buoyancy strength $G r / R e^{2}$ largely determines the base frequency of the oscillation. They also found that using larger diameter vortex generators and larger pitch space between the ribs increases wall heat transfer. Korichi et al. [7] studied heat transfer enhancement in self-sustained oscillatory flow in a grooved channel with oblique plates on the opposite wall at $250 \leqslant R e \leqslant 1000$ and analysed the fluid flow and heat transfer at different plate length, plate angle and Reynolds numbers. The presence of vortex generators at the upper surface was found to be a powerful mean to enhance the heat transfer compared to the basic grooved channel. For example, the heat transfer enhanced up 200\% for $R e=600$ and for a given geometric configuration of the oblique. Icoz and Jaluria [8] studied heat transfer enhancement in a rectangular channel with an aspect ratio of 6 with obstacles of various shapes at Reynolds numbers lower than 6000. The vortex promoters had circular, square, and hexagonal shapes, and were located, with several blockage ratios, ahead of two tandem heating sources. The circular promoter was found to be the best choice when the pressure loss is the main criteria concern. As the importance of the pressure drop is reduced relative to the heat transfer rates, hexagonal and square geometries were found to have better performances. Abbassi et al. [9] studied heat transfer enhancement due to triangular vortex promoter and reported 85\% increase in the time-averaged Nusselt number at a Reynolds number of 250. Nitin and Chhabra [10] considered a rectangular vortex promoter immersed in a non-Newtonian fluid. They reported heat transfer variations of the order of $10 \%$ and a strong sensitivity on the power law used to describe non-Newtonian behaviours. Meis et al. [11] investigated heat transfer enhancement in micro-channels caused by elliptical, rectangular, or triangular obstacles in a Reynolds number range between 600 and 1200. For all types of vortex promoters, they reported a heat transfer enhancement with a blockage ratio increase up to $1 / 2$. Among the various cross-sections examined, the triangular vortex generator produced the best heat transfer augmentation. They found performance improvement with aspect ratio decrease. Chattopadhyay [12] investigated convective heat transfer in a channel in the turbulent flow regime up to the Reynolds number of 40000 in the presence of a triangular obstacle and showed a heat transfer enhancement of about $15 \%$ compared to the plane channel. As expected, heat transfer augmentation was associated with pressure drop increase. However, performance analysis was not performed to compare the heat transfer augmentation and pressure loss penalty. Recently, Moussaoui et al. [13] applied the Lattice Boltzmann method to investigate fluid flow and heat transfer in a channel with a confined inclined $45^{\circ}$ square cylinder at $\operatorname{Re}<300$ and $\operatorname{Pr}=0.7$. Their results were presented in terms of streamline contours, isotherms, Strouhal number, Nusselt number and drag coefficient for various Reynolds numbers. The computed results showed that the presence of square cylinder significantly influences the fluid flow and causes an enhancement of heat transfer from the channel wall. Herman and Kang [14] have performed an experimental work to compare heat transfer enhancement in a grooved channel using a curved vane and a circular cylinder at the inlet. They compared their results with a simple grooved channel and two parallel plates in a Reynolds number range between 530 and 6200 . They found that each of the three methods offered some advantages in electronic cooling.

More recently, heat transfer enhancement by oscillating vortex generators has been the topic of several investigations. For example, Celik et al. [15] using Spectral Element Method studied heat transfer enhancement in a channel via a transversely oscillating adiabatic circular cylinder at a Reynolds number of 100 and in a Prandtl number range between 0.1 and 10 . The authors reported that cylinder oscillations with $75 \%$ of the natural vortex shedding frequency yields the best heat transfer augmentation with only $10 \%$ more power to pump the fluid compared to the case of a fixed cylinder. Yang [16] has carried out a similar investigation for a square obstacle and discussed the effect of amplitude, frequency, and maximum velocity. In the same oscillating amplitude of the bar, the heat transfer increment is decreased significantly as the maximum oscillating speed or the oscillating frequency of the bar is larger. Fu and Tong [17] carried out a numerical simulation of the effect of an oscillating cylinder on the heat transfer from heated blocks in a channel flow. Their results proved that heat transfer was remarkably enhanced as the oscillating frequency of the cylinder was in the lock-in region. A numerical study of convective heat transfer from a rotating cylinder with cross-flow oscillation has been done by Ghazanfarian and Nobari [18]. It was found that, in a similar manner to the fixed cylinder case, beyond a critical rotating speed, vortex shedding is mainly suppressed. Also, by increasing the non-dimensional rotational speed of the cylinder, both the Nusselt number and the drag coefficient decrease rapidly. In our last work [19], heat transfer enhancement in a channel via a rotationally oscillating cylinder was investigated. It was found that the maximum heat transfer was achieved when the oscillating frequency is $80 \%$ of the natural frequency of vortex shedding in presence of a stationary cylinder.

According to the author's knowledge, there is no research in which the effect of the distance from the cylinder to the lower wall on the vortex shedding phenomenon and heat transfer augmentation was investigated. Besides, just in few researches, the effect of the fluid type (Prandtl number) on the efficiency of the vortex generator has been studied. The main objective of the present study is to fill in these gaps. In addition, by calculating the pressure drop in the channel, we obtain a performance analysis at different Prandtl numbers. 


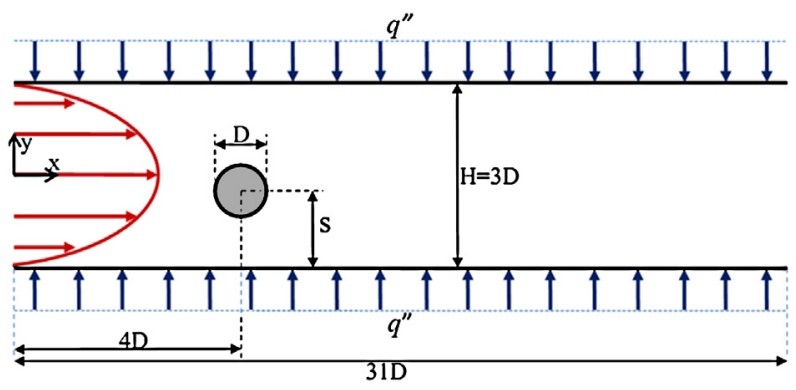

Fig. 1. Scheme of the computational domain.

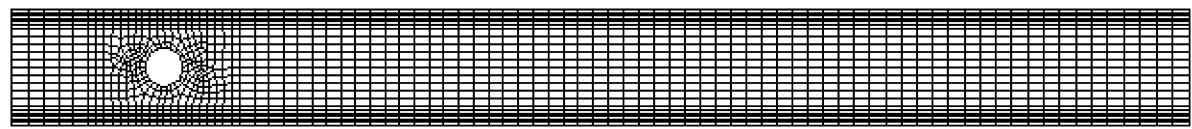

Fig. 2. Scheme of the generated mesh in the domain (the real mesh is 8 times finer than this one).

\section{Problem statement and formulation}

The side-view of the channel investigated in this study is shown in Fig. 1. An adiabatic circular cylinder with blockage ratio $D / H$ of $1 / 3$ is placed in the channel. The centre of the cylinder is located at a distance $s$ from the bottom wall and the distance of the obstacle centre from the inlet plane of the channel is $4 D$. The non-dimensional distance between the cylinder centre and bottom wall $(s / D)$ varies between 0.5 and 1.5 . For the highest value of gap spacing $(s / D=1.5)$, the centre of the cylinder coincides with the channel axis, whilst for the lowest value of $s / D=0.5$, the cylinder is attached to the bottom wall. The channel side along the lateral direction $(z)$ is assumed to be long. Thus, the three-dimensional effects due to side walls may be ignored and computations can thus be performed in two dimensions. For all simulations, the Reynolds number, based on the cylinder diameter, $D$, and the bulk velocity, is kept constant at Re=100, while the Prandtl number is varying from 0.1 to 10 .

For an unsteady incompressible flow, the conservation laws of mass, momentum and energy in tensor notation are written as:

Continuity:

$$
\frac{\partial U_{j}}{\partial x_{j}}=0
$$

Momentum:

$$
\frac{\partial U_{i}}{\partial t}+\frac{\partial\left(U_{j} U_{i}\right)}{\partial x_{j}}=-\frac{1}{\rho} \frac{\partial P}{\partial x_{i}}+v \frac{\partial^{2} U_{i}}{\partial x_{j} \partial x_{j}}
$$

Energy:

$$
\frac{\partial T}{\partial t}+\frac{\partial\left(U_{j} T\right)}{\partial x_{j}}=\alpha \frac{\partial^{2} T}{\partial x_{j} \partial x_{j}}
$$

where $\rho, v$ and $\alpha$ are, respectively, the density, the kinematic viscosity, and the thermal diffusivity of the fluid.

As shown in Fig. 1, a fully developed flow with parabolic velocity profile and maximum velocity $1.5 \bar{U}$ enters the channel with a constant temperature $T=0.0$. No slip-boundary condition is applied on any solid boundary. Zero heat flux thermal boundary conditions are applied on the cylinder surface and constant heat flux thermal boundary conditions on the channel walls $\left(q^{\prime \prime}=1.0\right)$. Zero Neumann boundary conditions are applied for both velocity and temperature at the channel outlet, that is located $31 \mathrm{D}$ away from the inlet. Computations of the present study are obtained using OpenFOAM code. The pressure field is linked to the velocity field using PISO algorithm. A second-order upwind scheme is employed for approximating nonlinear convective terms in momentum and energy equations, while temporal derivatives are discretized using the second order Crank-Nicolson scheme. A hybrid structured-unstructured mesh consisting of around $4 \times 10^{4}$ triangular and quadrilateral nodes is employed for simulations. A series of grid-independency tests have been carried out to assess the accuracy of the computations. The results of these studies have shown that the grids used here are sufficiently fine to produce grid-independent results (see also Fig. 2). 

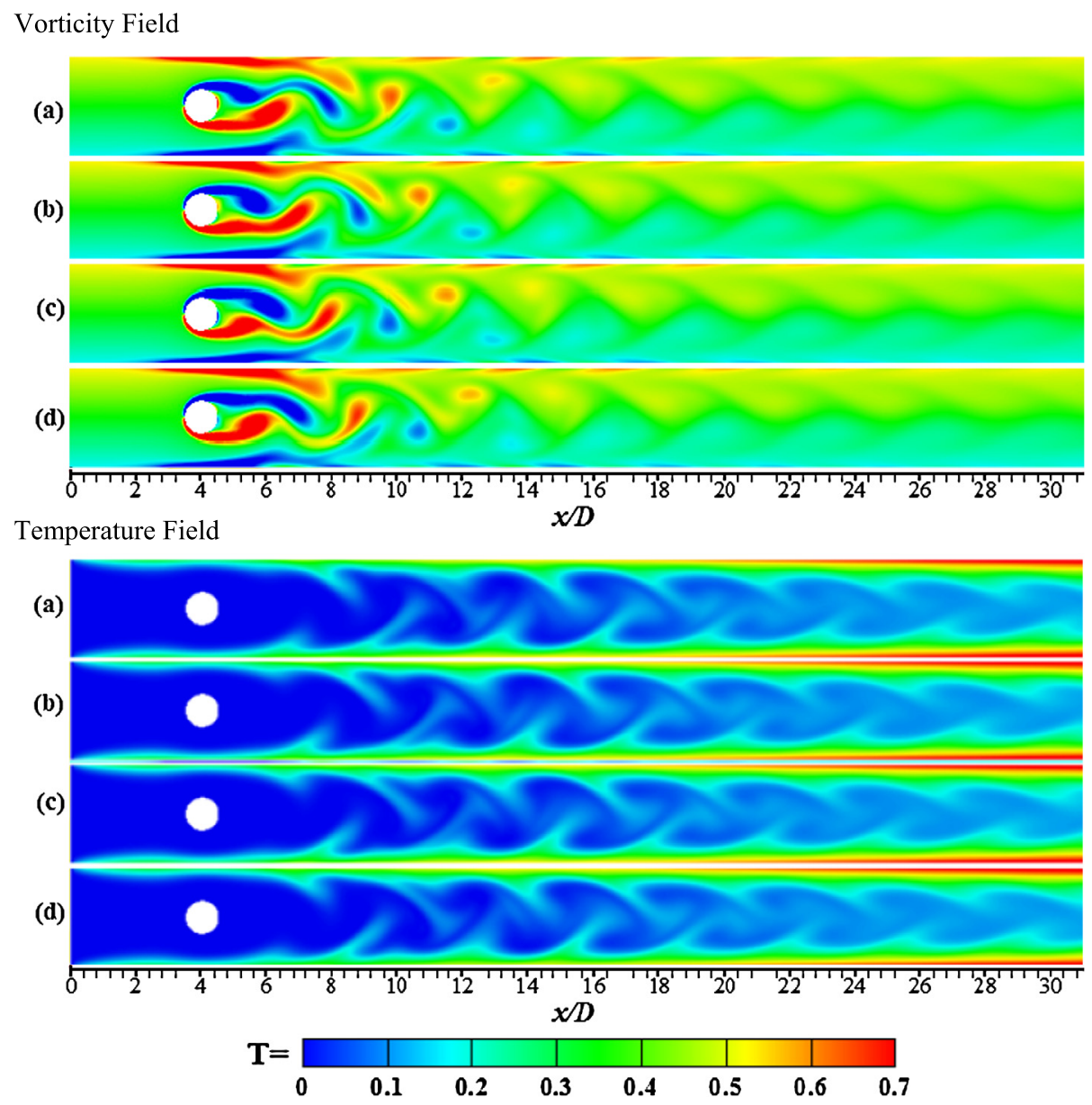

Fig. 3. (Colour online.) Instantaneous vorticity and temperature contours for $s / D=1.5$ and $\operatorname{Pr}=1.0$ at times $t=0.0$ (a), $t=\tau / 4$ (b), $t=\tau / 2$ (c), and $t=3 \tau / 4(\mathrm{~d})$.

\section{Results and discussion}

In the following, the results obtained for various gap spacings are presented and discussed. The first attention is focused on the local heat transfer distribution, and then average heat transfer levels will be presented. Finally, performance analysis will be provided.

\subsection{Local Nusselt number distribution}

For $s / D=1.5$, representing the case where the cylinder centre is positioned on the channel axis, the computed instantaneous vorticity and temperature contours at four instants of the vortex shedding period $(\tau)$ are shown in Fig. 3. As can be observed, negative and positive vortices are shed alternatively from the upper and lower surfaces of the cylinder, creating a Karman vortex street downstream from the cylinder. The corresponding value of the Strouhal number, obtained by monitoring the pressure variation at a point downstream from the cylinder, is 0.36 , which is almost twice the value for a similar flow around an unconfined cylinder [20]. As can be seen in Fig. 3, the vortex patterns and temperature contours are correlated. The braid-like temperature contours in the downstream and corrugated structure of the thermal boundary layers are due to the vortex dynamics that transport the hot fluid on channel walls to the main stream, and vice versa.

To assess the results for time-periodic flow, the instantaneous Nusselt number on the upper and the lower walls of the channel are calculated. On any surface, the local Nusselt number, $N u(x, t)$, is defined as:

$$
N u(x, t)=\frac{q^{\prime \prime} H}{\kappa\left(T_{\mathrm{w}}-T_{\mathrm{b}}\right)}
$$

where $\kappa$ is the thermal conductivity, $q^{\prime \prime}$ is the wall heat flux, $T_{\text {wall }}$ is the wall temperature and $T_{\mathrm{b}}$ is the bulk temperature given by: 


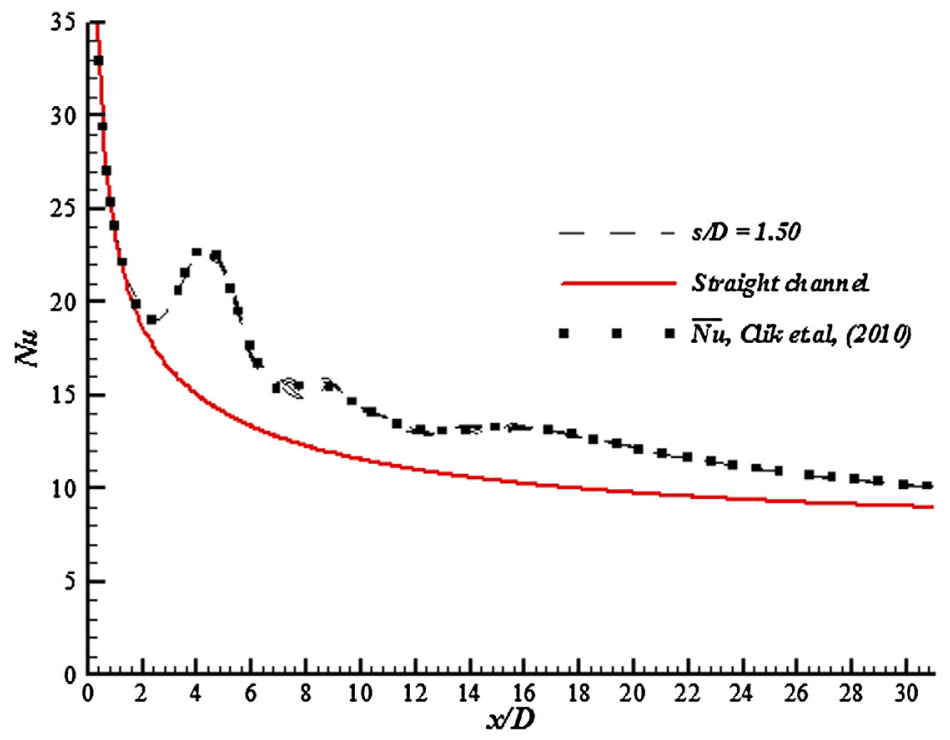

Fig. 4. Variation of the instantaneous Nusselt number in the stream-wise direction within a period of vortex shedding for $s / D=1.5$ and $\operatorname{Pr}=1.0$.

$$
T_{\mathrm{b}}=\int_{-H / 2}^{H / 2} U T \mathrm{~d} y / \int_{-H / 2}^{H / 2} U \mathrm{~d} y
$$

For time-periodic flows, we obtained the time-averaged Nusselt number along the channel, $N u(x)$, by integrating the local Nusselt number, $N u(x, t)$, over the vortex shedding period, $\tau$, as follows:

$$
\overline{N u}(x)=\frac{1}{\tau} \int_{0}^{\tau} N u(x, t) \mathrm{d} t
$$

As shown in Fig. 3, due to time-periodicity, instantaneous asymmetry in the flow field and temperature profiles induced by the vortex patterns repeat cyclically. As a result, for $s / D=1.5$, the instantaneous $N u$ at a stream-wise location, $x$, on the upper wall, is observed half a period later at the same $x$-location on the lower wall. Therefore, the time-averaged Nusselt number obtained via Eq. (6) is representative of the behaviour of both channel surfaces. The time-dependent Nusselt numbers along the channel at different instances within a period are plotted in Fig. 4 for $s / D=1.5$ and compared with the Nusselt number distribution of a thermally developing flow through a plane channel under a constant heat flux thermal boundary condition at the same Reynolds number and with those obtained by [15] using the spectral method. It is clearly seen that the results of the present computations are in excellent agreement with those obtained using a highly accurate spectral element method, which further supports the accuracy of the numerical scheme employed in the present work. As expected, for the straight channel, the Nusselt number decreases gradually to approach the fully developed value of $8.23[21]$.

In presence of the cylinder, a significant heat transfer enhancement at cylinder downstream is observed. Specifically, the Nusselt number peaks locally at $x / D=4.0$ because of the flow acceleration due to the blockage effect. There is a relatively smaller local peak at $x / D=8.0$, which is induced by vortex shedding at the end of the formation region (see vorticity contours in Fig. 3). It appears that heat transfer enhancement depends on the ability of vortices to entrain the cold fluid in the middle of the channel towards the walls, and removal of heated fluid away from the channel walls.

To examine the effects of gap spacing on fluid flow characteristics, vorticity contours for smaller gap spacing of $s / D=$ 1.25 and $s / D=1.00$ are presented in Fig. 5. Comparison of vorticity contours obtained for $s / D=1.25$ with those shown in Fig. 3 for $s / D=1.5$ indicates that stronger vortices are now shed from the upper side of the cylinder, whilst the lower side vortices become notably weaker. This feature is expected since for $s / D=1.25$, the cylinder surface is at a larger distance from the upper wall than from the bottom wall. Thus, the lower wall damps the vortices more than the upper wall. As the cylinder approaches further towards the bottom wall (i.e. $s / D=1.00$ ), the strength of vortices seems to be further diminished and the lower vortices completely disappear. This demonstrates the suppression of the vortex shedding phenomena by the bottom wall. As shown in Fig. 6, further displacement of the obstacle towards the bottom wall leads to a complete suppression of the vortex shedding and the establishment of a steady flow.

To further demonstrate how the dynamic field influences the wall heat transfer, distributions of time-averaged Nusselt numbers (Eq. (6)) along the top and bottom walls of the channel for various $s / D$ are presented in Fig. 7 and compared with the plane channel Nusselt number distribution. For $s / D=1.50$ and 1.25 , it is observed that Nusselt number distributions 



Fig. 5. (Colour online.) Instantaneous vorticity contours for $s / D=1.25$ and 1.00 at times $t=0.0$ (a), $t=\tau / 4$ (b), $t=\tau / 2$ (c), and $t=3 \tau / 4$ (d).

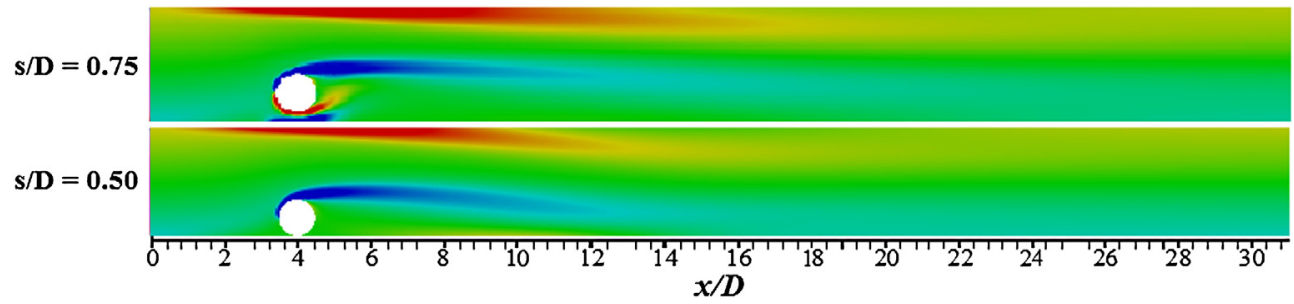

Fig. 6. (Colour online.) Steady-state vorticity contours for $s / D=0.75$ and $s / D=0.5$.

are much similar (i.e. the Nusselt number first follows a plane channel Nusselt number distribution and subsequently experiences two peaks and one crest in the zone downstream from the cylinder). It is noted that for both cases, the Nusselt number levels along the walls are higher than those obtained for the plane channel. As demonstrated above, these variations are consistent with the flow field results shown in Figs. 3 and 5.

For $s / D=1.00$, the Nusselt number variations along both heated walls become smoother. In the immediate region downstream from the cylinder, it is noted that the bottom wall Nusselt number values become lower than those for the plane channel. Suppression of vortex shedding for $s / D=0.75$ and 0.50 results in much lower heat transfer levels along both walls and especially along the lower wall. For $s / D=0.5$, the fluid in front and behind the cylinder is stagnant. This leads to very low heat transfer coefficients on the lower wall and thus formation of hot spots. It is seen that the upper wall Nusselt number distribution is not sensitive to $s / D$ as that on the lower wall. For $1.0<s / D<1.5$, where an unsteady flow is established, there are not major differences in Nusselt numbers. On the other hand, for $s / D=0.75$ and 0.5 , the second small pick of the Nusselt number at $x / D=8.9$ has now disappeared, due to the complete suppression of the vortex shedding and the establishment of a steady flow.

\subsection{Averaged Nusselt number}

In Fig. 8, averaged Nusselt numbers along the channel walls for $\operatorname{Pr}=0.1,1.0$ and 10.0, obtained from integration of local Nusselt number (Eq. (7)), are presented. 



Fig. 7. Stream-wise variation of the time-averaged Nusselt number along the lower and upper walls of the channel $(\operatorname{Pr}=1.0)$.

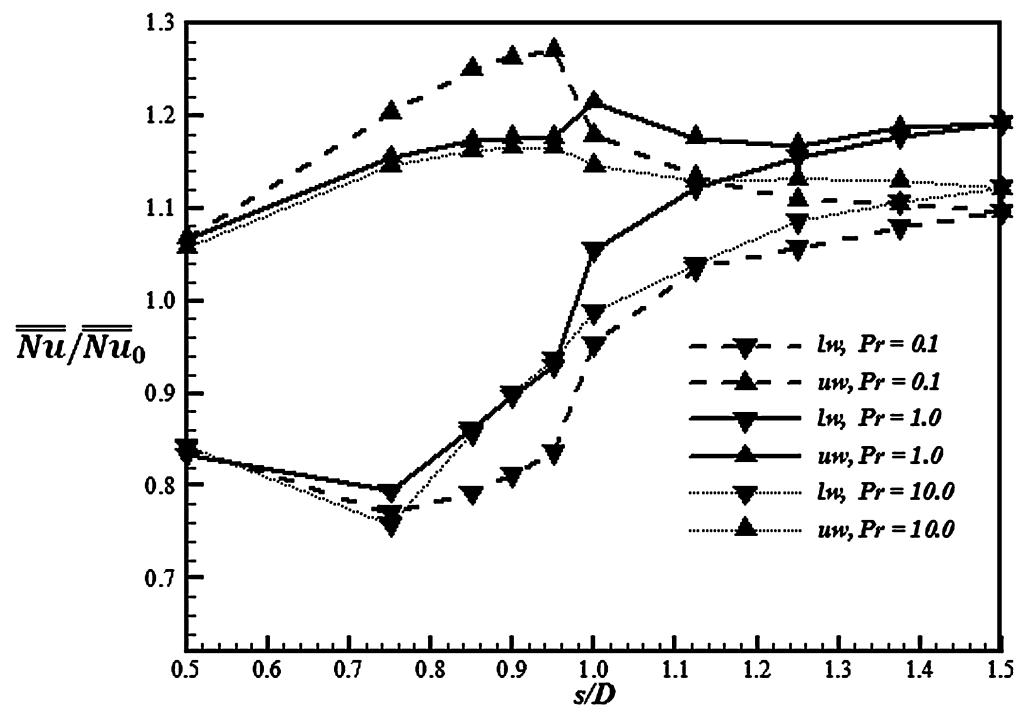

Fig. 8. Effects of the gap spacing on the averaged Nusselt number.

$$
\overline{\overline{N u}}=\frac{1}{L} \int_{0}^{L} \overline{N u}(x) \mathrm{d} x
$$

To show the overall heat transfer enhancement, the averaged Nusselt numbers are normalized with the thermally developing plane channel averaged Nusselt number. It is seen that for $s / D=1.5$ and $\operatorname{Pr}=0.1$, heat transfer enhancement on both walls is around $20 \%$. As the cylinder approaches the bottom wall, consistently with flow field predictions and also Nusselt number variations in Fig. 7, the bottom-wall averaged Nusselt number gradually drops to 0.85 , while experiencing a turning point at $s / D \approx 1.0$. This turning point could be due to the transition from an unsteady flow to a steady flow at gap spacing values lower than $s / D \approx 1.0$. It is seen that the averaged Nusselt number along the upper wall slightly increases with a reduction of $s / D$ up to $s / D \approx 1.0$, which is in agreement with flow field predictions. With further reduction of $s / D$, the upper wall Nusselt number starts to decrease, which agrees with the flow field results shown in Figs. 3 and 5 . It is seen that the overall trend of the Nusselt number distributions for $\operatorname{Pr}=1$ and 10 is similar to that of $\operatorname{Pr}=0.1$. It is noted that normalized averaged Nusselt distributions become smoother as $\operatorname{Pr}$ increases. Also this figure shows that, for $s / D>1.0$, normalized Nusselt number levels of $\operatorname{Pr}=1$ are higher than those for $\operatorname{Pr}=0.1$ and 10.0. Fig. 9 shows the effects of gap spacing on the channel pressure loss. Here again, the computed pressure loss for the channel with obstacle is normalized with the plane channel pressure drop $\Delta P_{0}$. It is seen that the highest pressure loss occurs for $s / D=1.5$. Reduction of $s / D$ 


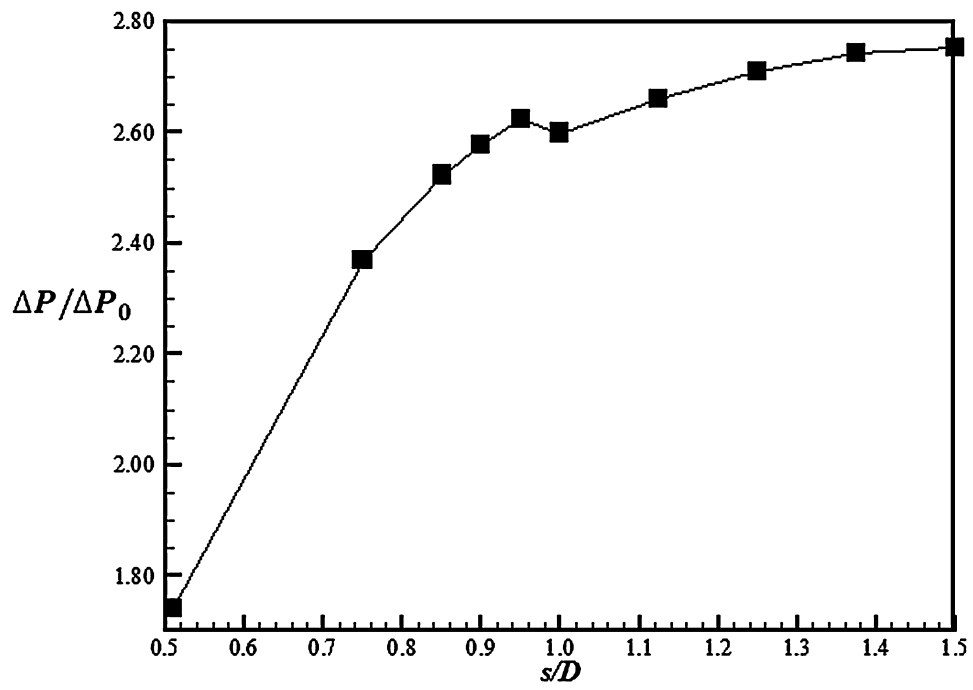

Fig. 9. Effects of the gap spacing on the channel pressure loss.

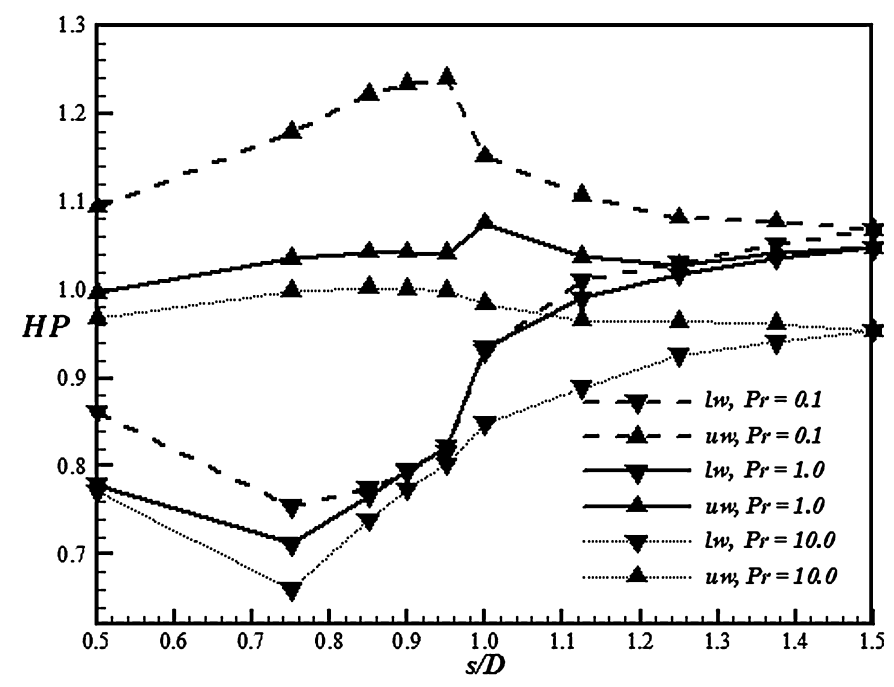

Fig. 10. Thermal effectiveness versus gap spacing.

gradually decreases the pressure drop up to $s / D \approx 1.0$. Further reduction of $s / D$ causes the pressure drop to rise slightly and then to start to decrease again. As mentioned above, this could be due to a change from an unsteady flow to a steady flow as the cylinder approaches the lower wall.

\subsection{Performance analysis}

To find out the usefulness of obstacle insertion, the required pumping power for each $s / D$ is computed and applied along the straight channel. Using basic concepts of laminar flow [22] through a 2D channel, the corresponding inlet velocity for a given pumping power is obtained from:

$$
V^{*}=\sqrt{\text { Power } \times \frac{H}{12 \mu L}}
$$

Fig. 10 demonstrates the variation of $H P=\overline{\overline{N u}} / \overline{\overline{N u^{*}}}$ (the ratio of the averaged Nusselt number in the presence of the obstacle to the averaged Nusselt number in a straight channel for a given pumping power) versus $s / D$. HP values greater than 1 indicate that the insertion of an obstacle to cooling is beneficial to average heat transfer considering pressure drop penalty. As can be seen in Fig. 10, for gas flows with Prandtl numbers between 0.1 and 1, the use of an obstacle to overall heat transfer enhancement is beneficial. This could due to the fact that for identical Reynolds numbers, the thermal boundary 


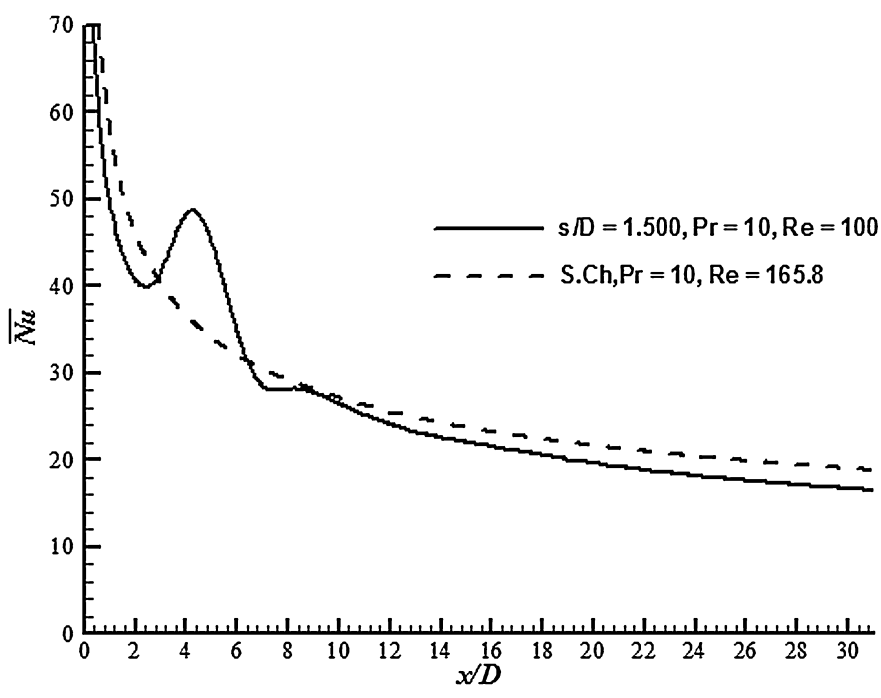

Fig. 11. Nusselt distribution along the channel by using the same power.

layer of gases is thicker for gases than for liquids, and consequently vortex shedding can more effectively mix the bulk cold fluid with the hot fluid adjacent to the channel wall. For $\operatorname{Pr}=0.1$, it is clear that the use of an obstacle is useful $(H P>1)$ for both walls when $s / D>1.25$. As the Prandtl number increases, the critical $s / D$ for useful performance increases and for $\operatorname{Pr}=10.0$, there is no gap spacing, which provides $H P>1$ for both walls. It is evident that at each gap spacing, the presence of obstacle is more effective on the cooling of the upper wall and thus, when the cooling of the upper wall is more important, the $s / D$ value giving the maximum HP may be considered.

Although application of an obstacle for high-Pr-number fluids appears not to be beneficial to average heat transfer enhancement, picks in the local Nusselt number distribution can be useful when local cooling is required. To demonstrate this further, in Fig. 11, for $\operatorname{Pr}=10$ and $s / D=1.5$, the Nusselt number distributions along the channel in the presence of the obstacle and $R e=100$, and in the absence of the obstacle but with a similar pumping power (giving $R e=165.8$ ) are presented. As seen in this figure, though the average $\mathrm{Nu}$ for the plane channel is higher, the insertion of the cylinder causes a local increase in heat transfer at $3<x / D<6.2$, which could be valuable when cooling in this region is needed.

\section{Conclusions}

In the present paper, the effect of gap spacing between the cylinder and the lower wall on heat transfer augmentation and pressure loss in a channel is investigated numerically. Performance analysis has also been performed to find the optimum cylinder position for maximum heat transfer enhancement and reasonable pressure drop. Simulation results have revealed that heat transfer increases from channel walls as a result of flow acceleration and vortex shedding phenomena. By moving the cylinder towards the lower wall, flow and thermal fields gradually tend to become steady and pressure drop decreases. Considering pressure drop and average cooling rate, the results of the present study show that using the cylinder is useful for gas flows. For fluids with Prandtl numbers greater than 1, the insertion of the obstacle in the channel is not recommended, though it can provide local wall heat transfer increase. In other words, using the vortex generator is recommended for gas flows $(0.1 \leqslant \operatorname{Pr} \leqslant 1)$.

\section{References}

[1] B. Rezaie, M.A. Rosen, District heating and cooling: Review of technology and potential enhancements, Appl. Energy 93 (2012) 2-10.

[2] I. Nkurikiyimfura, Y. Wang, Z. PanHeat, Transfer enhancement by magnetic nanofluids-A review, Renew. Sustain. Energy Rev. 21 (2013) 548-561.

[3] S. Laohalertdecha, P. Naphon, S. Wongwises, A review of electrohydrodynamic enhancement of heat transfer, Renew. Sustain. Energy Rev. 11 (2007) $858-876$.

[4] M. Rahnama, H.H. Moghaddam, Numerical investigation of convective heat transfer in unsteady laminar flow over a square cylinder in a channel, Heat Transf. Eng. 26 (2005) 21-29.

[5] T.K. Yrum, X. Qiu, S. Acharya, Heat transfer enhancement in a ribbed duct using vortex generators, Int. J. Heat Mass Transf. 36 (1993) 3497-3508.

[6] Q. Wang, Y. Jaluria, Unsteady mixed convection in a horizontal channel with protruding heated blocks and a rectangular vortex promoter, Phys. Fluids 14 (2002) 2113-2137.

[7] A. Korichi, L. Oufer, G. Polidori, Heat transfer enhancement in self-sustained oscillatory flow in a grooved channel with oblique plates, Int. J. Heat Mass Transf. 52 (2009) 1138-1148.

[8] T. Icoz, Y. Jaluria, Design optimisation of size and geometry of vortex promoter in a two-dimensional channel, J. Heat Transf. 128 (2006) $1081-1092$.

[9] H. Abbassi, S. Turki, S.B. Nasrallah, Numerical investigation of forced convection in a plane channel with a built-in triangular prism, Int. J. Therm. Sci. 40 (2001) 649-658.

[10] S. Nitin, R.P. Chhabra, Non-isothermal flow of a power law fluid past a rectangular obstacle in a channel: Drag and heat transfer, Int. J. Eng. Sci. 43 (2005) 707-720. 
[11] M. Meis, F. Varas, A. Velázquez, J.M. Vega, Heat transfer enhancement in micro-channels caused by vortex promoters, Int. J. Heat Mass Transf. 53 (2010) 29-40.

[12] H. Chattopadhyay, Augmentation of heat transfer in a channel using a triangular prism, Int. J. Therm. Sci. 46 (2007) 501-505.

[13] M.A. Moussaoui, M. Jami, A. Mezrhab, H. Naji, MRT-lattice Boltzmann simulation of forced convection in a plane channel with an inclined square cylinder, Int. J. Therm. Sci. 49 (2010) 131-142.

[14] C. Herman, E. Kang, Comparative evaluation of three different heat transfer enhancement strategies in a grooved channel, Heat Mass Transf. 37 (2001) 563-575.

[15] B. Celik, M. Raisee, A. Beskok, Heat transfer enhancement in a slot channel via a transversely oscillating adiabatic circular cylinder, Int. J. Heat Mass Transf. 53 (2010) 626-634.

[16] S.J. Yang, Numerical study of heat transfer enhancement in a channel flow using an oscillating vortex generator, Heat Mass Transf. 39 (2003) $257-265$.

[17] W.S. Fu, B.H. Tong, Numerical investigation of heat transfer characteristics of the heated blocks in the channel with a transversely oscillating cylinder, Int. J. Heat Mass Transf. 47 (2004) 341-351.

[18] J. Ghazanfarian, M.R.H. Nobari, A numerical study of convective heat transfer from a rotating cylinder with cross-flow oscillation, Int. J. Heat Mass Transf. 52 (2009) 5402-5411.

[19] A. Beskok, M. Raisee, B. Celik, B. Yagiz, M. Cheraghi, Heat transfer enhancement in a straight channel via a rotationally oscillating adiabatic cylinder, Int. J. Therm. Sci. 58 (2012) 61-69.

[20] B. Celik, U. Akdag, S. Gunes, A. Beskok, Flow past an oscillating circular cylinder in a channel with an upstream splitter plate, Phys. Fluids 20 (2008) 103-603.

[21] F.P. Incropera, D.P. Dewitt, Fundamentals of Heat and Mass Transfer, John Wiley and Sons, New York, 1990.

[22] R.W. Fox, A. McDonald, P.J. Pritchard, Introduction to Fluid Mechanics, John Wiley and Sons, New York, 2004. 